\title{
The Mantle of Advanced Glycation End Products in Micro-and Macrovascular Complications of Type 2 Diabetes Mellitus
}

\author{
Attya Bhatti*, Nida Ali Syed and Peter John \\ Department of Healthcare Biotechnology, National University of Sciences and Technology, Pakistan
}

Submission: June 14, 2017; Published: June 30, 2017

*Corresponding author: Attya Bhatti, Department of Healthcare Biotechnology, National University of Sciences and Technology, Pakistan, Tel: +92-51-9085-6128; Email: attyabhatti@gmail.com

\begin{abstract}
Type 2 Diabetes Mellitus is a multifactorial disease marked by chronic hyperglycemia and insulin resistance. Its associated vascular complications are a major contributor to mortality globally. These vascular complications are frequently implicated to be an offshoot of high levels of advanced glycation end products which are produced due to a persistent hyperglycemic and oxidative stress state. This review summarizes the role of advanced glycation end products in the pathogenesis of micro- and macrovascular complications of type 2 diabetes mellitus.
\end{abstract}

Keywords: Type 2 diabetes mellitus; Advanced glycation end products; Diabetic nephropathy; Diabetic neuropathy; Cardiovascular disease; Diabetic retinopathy.

\section{Introduction}

\section{Type 2 Diabetes Mellitus}

Type 2 Diabetes Mellitus is a multifactorial disorder that occurs because of a complex interplay between genetic predisposition and lifestyle choices as the two primary causative factors. It is characterized by chronic inflammation, insulin resistance, oxidative stress and hyperglycemia. Chronic state of hyperglycemia often results in the formation of Advanced Glycation End products, hereby abbreviated as AGEs via the infamous Maillard reaction. The Maillard reaction is defined as formation of adducts between reactive carbonyls in glucose, fructose, and their metabolites, such as methylglyoxal or deoxyglucosone, with amino groups in protein, DNA, and lipids. This reaction has been implicated as a root cause of several evils in diabetes associated micro- and macrovascular complications [1].

Advanced Glycation End Products are frequently referred to as glycotoxins; whose formation is induced by nonenzymatic glycemic and oxidative stress reactions [2]. These are a heterogenous group of biological entities formed via a nonenzymatic post-translational modification reaction between reducing sugars and the amino groups of proteins, nucleic acids and lipids [3]. AGEs are formed by the Maillard reaction was first described in 1912, by French scientist Louis Camille Maillard. This is a multistep process, initiated by the reversible reaction between the carbonyl group of a reducing sugar and terminal amino group of a protein, lipid or nucleic acid, resulting in the formation of a Schiff base. These further undergo irreversible rearrangements to form more stable ketoamines hereby referred to as the Amadori products; a putative example of which is HbA1c.These products undertake further structural rejoinders via oxidation, condensation, dehydration over the span of days to weeks and give way to irreversibly cross-linked, fluorescent microprotein derivatives known as AGEs [4,5]. These products persist in diabetic vessels for long time despite improved glycemic control and undergo slow degradation Figure 1.

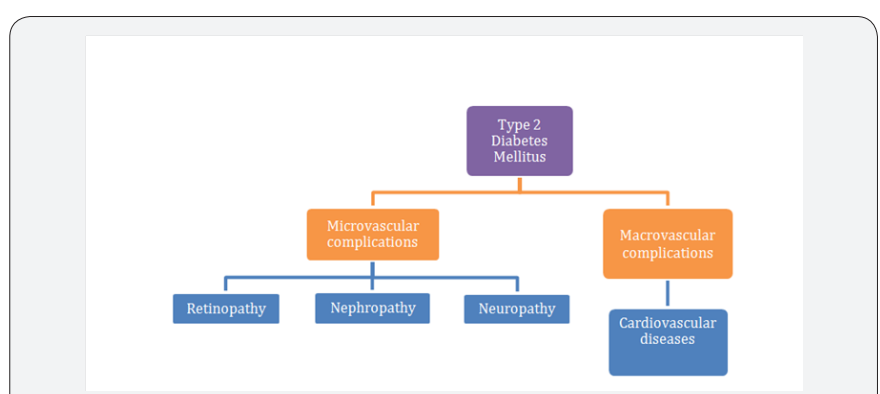

Figure 1: Vascular complications of Type 2 Diabetes Mellitus. 


\section{Current Research in Diabetes \& Obesity Journal}

\section{Pathophysiological role of AGEs in vascular complications in Diabetes}

In the context of type 2 diabetes mellitus, accelerated formation and accumulation of AGEs has been implicated in the onset of diabetic microvascular complications which are summarised below.

Diabetic retinopathy: Diabetic Retinopathy is one of the foremost microvascular complications in T2DM and the leading cause for acquired blindness in individuals of abstruse age/young adults. It starts with retinal microvascular cells being damaged due to hyperglycemia. Subsequent loss of pericytes leads to enhanced vascular permeability which leads to microvascular occlusion in the retina $[6,7]$. AGEs have been implicated in the onset and progression of microvascular disease in DM. Levels of endothelial cell specific mitogen VEGF in ocular fluid have been reported in various clinical studies to positively correlate with the amount of neovascularization in diabetic retinopathy [8]. AGEs accumulate in retinal pericytes during diabetes and adversely affect their function and survival [9]. Various studies have shown that AGEs accumulation causes apoptosis of retinal pericytes and its interaction with RAGE induces the expression of VEGF, DNA synthesis and angiogenesis which are all regarded as hallmarks of proliferative retinopathy [10]. AGE induced damage to pericytes predisposes vessels to angiogenesis, thrombogenesis and endothelial cell injury; which results in an overt clinical expression of diabetic retinopathy increased [11]. AGEs have been shown to stimulate angiogenesis by inducing growth and tube formation of microvascular endothelial cells via interaction with RAGE and subsequent VEGF expression [12]. AGEs have also demonstrated an enhanced leukocyte adhesion to cultured retinal microvascular endothelial cells by induction of the expression of ICAM-1 (intracellular cell adhesion molecule-1), which leads to leukostasis and blood-retinal barrier dysfunction as shown in various in vivo experiments [13-15].

Diabetic nephropathy: Nephropathy is one of the most common complications of T2DM. Increased glomerular basement membrane thickness, a decreased glomerular filtration rate, and an expanded mesangial volume are regarded as hallmarks of nephropathy [16]. Diabetes induced alterations in the physical and biochemical properties of the glomerular basement membrane results in proteinuria. AGEs have been implicated in the disruption of glomerular homeostasis as their accumulation in mesangial cells induces apoptosis and inhibits cell growth. Mesangial cells represent a key anatomical component of glomerulus, providing structural support for capillary tufts and modulating glomerular filtration via smooth muscle activity $[17,18]$. Secretion of VEGF and monocyte chemoattractant protein-1 (MCP-1) is also stimulated by AGEs, which results in hyperfiltration and microalbuminuria thus leading to the early phase of diabetic nephropathy [10]. Furthermore, serum levels of AGEs were also elevated in diabetic patients with nephropathy than in diabetic patients without clinically evident nephropathy [19]. These findings are suggestive of the fact that AGEs impact mesangial cells in the same manner as they affect pericytes and vascular wall damage is the stepping stone for all diabetic vascular complications. Over expression of RAGE in diabetic mice resulted in progressive glomerulosclerosis and renal dysfunction where as inactivation of RAGE in a mouse model of diabetic nephropathy suppressed kidney enlargement, increased glomerular cell number, induced mesangial expansion, advanced glomerulosclerosis, increased albuminuria, and increased serum creatinine levels compared with wild-type diabetic mice [20,21]. In the latter study, low molecular weight heparin treatment specifically prevented albuminuria, increased glomerular cell number, mesangial expansion, and glomerulosclerosis by acting as an antagonist to RAGE [20]. Recent studies have shown that RAS (renin-angiotensin system) inhibitors such as telmisartan or olmesartan can potentially inhibit AGEs evoked inflammatory responses in endothelial cells by downregulating RAGE expression. There by potentially preventing of diabetic vascular complications [22,23].

Diabetic neuropathy: Diabetes Mellitus is a key cause of peripheral neuropathy, which typically presents as distal symmetrical polyneuropathy [19]. Key pathological developments in human diabetic nerves include fiber loss, axonal degeneration and demyelination, and microangiopathic changes $[24,25]$. AGEs have been detected in sural, peroneal, and saphenous nerves of human diabetic subjects in the perineurium, endothelial cells and pericytes of endoneurial microvessels as well as in myelinated and unmyelinated fibers [26]. Accumulation of AGEs in the nerves of diabetes patients and the inhibition of AGEs formation by anti-glycation agents improved the neuropathic changes suffered by experimental diabetic rat model [27]. However, the pathologic mechanisms behind the actions of AGEs in diabetic neuropathy are poorly understood. AGEs have been demonstrated to affect the viability, replication, and the production of proinflammatory cytokines such as tumor necrosis factor- $\alpha$ (TNF- $\alpha$ ) and interleukin-1 $\beta$ (IL$1 \beta$ ) in Schwann cells [28]. This toxic behavior of AGEs has also been observed in neuronal, vascular, and mesangial cells $[10,29]$.

\section{Cardiovascular diseases}

AGE modifications of proteins frequently involve cross linking of proteins which has been implicated in vascular and myocardial stiffness and deterioration of structural integrity and physiological functioning of various organ systems in the context of isolated systolic hypertension and diastolic heart failure [30]. Several in vitro, in vivo and epidemiological studies have declared atherosclerosis to be an intrinsically inflammatory disease of the heart [31]. Activation of the AGE-RAGE pathway leads to the generation of oxidative stress which subsequently activates NF-kB signaling pathway in vascular wall cells. This sequence of events promotes atherosclerosis and inflammation promoting genes' expression which contributes to the development and progression of cardiovascular complications in diabetes $[11,32,33]$. Nitric oxide (NO) is a potent endogenous vasodilator having anti-inflammatory, anti-thrombotic anti-proliferative 
and anti-arthrogenic properties [34]. AGEs have been shown to inhibit endothelial NO synthase and concomitantly stimulate the production of peroxynitrite; a reactive intermediate and toxic product of NO's reaction with superoxide anion. AGE-RAGE interaction also stimulates the production of an endogenous inhibitor of endothelial NO synthase, asymmetric dimethyl arginine (ADMA) that is expressed in endothelial, renal mesangial and renal proximal tubular cells $[35,36]$. ADMA has been recently recognized as a potent biomarker of CVD and chronic kidney disease progression and could also be involved in cardiorenal complications in diabetes [5,37]. In diabetic patients, AGE modification impairs the plasma clearance of low density lipoprotein (LDL) and converts it into a more atherogenic and redox-sensitive mitogen activated protein kinase (MAPK) activator [38].

AGEs have also been implicated in the reduction of adenosine triphosphate-binding membrane cassette transporter A1 (ABCA1) and ABCG1 levels in THP-1 cells that inhibits cholesterol efflux from THP-1 macrophages to Apolipoprotein AI and HDL cholesterol respectively. This cycle of events implicates the involvement of AGE-RAGE axis in impaired reverse translocation of cholesterol in diabetes and accelerated formation of foam cells in atherosclerotic lesions $[39,40]$. AGEs promote thrombogenesis by activating as well as aggregating platelets and by enhancing the expression of tissue factor, which leads to thrombus formation. Recent studies have also shown that AGEs potentiate thromb in or factor Xa- mediated endothelial and renal cell damages via up regulation of protease-activated receptor-1 and -2 [41-43]. AGEs' interaction with their respective RAGE inhibits prostacyclin production and induce plasminogen activator inhibitor-1 generation in endothelial cells [44]. Therefore, it can be stated conclusively that AGEs possess the ability to stimulate platelet aggregation and fibrin stabilization, resulting in a predisposition to thrombogenesis and promotion of vascular injury in diabetes. AGE-induced pathological neovascularization of atherosclerotic plaques is often mediated by an ischemia and hypoxia mediated upregulation of VEGF [45].

This triggers pathological angiogenesis which contributes to plaque growth and instability within the atherosclerotic plaques in diabetes [5]. Endothelial cell dysfunction and decreased endothelial progenitor cell (EPC) function is another hallmark of increased risk of cardiovascular complications in diabetic patients [46]. AGEs are known to enhance apoptosis and suppress migration and tube formation of late EPCs by interacting with RAGE and subsequent downstream suppression of Akt and COX-2 [47]. This modification impairs vascular repair by inhibiting EPC adhesion, spread and migration via glycation of Arg-Gly-Asp motif of fibronectin [48]. Vascular calcification in atherosclerosis is often mediated by AGEs by means of osteoblastic differentiation of pericytes. Activation of RAGE inhibits myocardin-dependent smooth muscle cell (SMC) gene expression and induces osteogenic differentiation of vascular SMCs through Notch/Msx2 induction thus being involved in vascular calcification as well [49]. AGEs have been implicated in the induction of oxidative stress that subsequently induces SMC proliferation via activation of NADPH oxidase. AGE-RAGEinduced extracellular signal related kinase activation is reported to increase $\mathrm{Na}+\mathrm{H}+$ exchanger- 1 activity, which leads to a decrease in intracellular $\mathrm{H}+$ and subsequently promotes a cellcycle progression and SMC proliferation [50].

\section{Conclusion}

Advanced Glycation End Products have been established to play a causative role in the onset of type 2 diabetes mellitus as well as its associated co-morbidities such as diabetic nephropathy, diabetic neuropathy, diabetic retinopathy and cardiovascular disease. This role is triggered by the chronic state of hyperglycemia that is accompanied by inflammation and oxidative stress and triggers multiple downstream signaling pathways that result in various micro- as well as macrovascular complications in diabetic patients.

\section{References}

1. Ott C, Jacobs K, Haucke E, Santos AN, Grune T, et al. (2014) Role of advanced glycation end products in cellular signaling. Redox Biol 2: 411-429.

2. Vlassara H, Cai W, Crandall J, Goldberg T, Oberstein R, et al. (2002) Inflammatory mediators are induced by dietary glycotoxins, a major risk factor for diabetic angiopathy. Proc Natl Acad Sci 99(24): 1559615601.

3. John WG, Lamb EJ (1993) The Maillard or browning reaction in diabetes. Eye 7(2): 230-237.

4. Méndez JD, Xie J, Aguilar-Hernández M, Méndez-Valenzuela V (2010) Trends in advanced glycation end products research in diabetes mellitus and its complications. Mol Cell Biochem 341(1-2): 33-41.

5. Yamagishi Si, Nakamura N, Suematsu M, Kaseda K, Matsui T (2015) Advanced Glycation End Products: A Molecular Target for Vascular Complications in Diabetes. Mol Med 21(1): S32-S40.

6. Frank RN (1991) On the pathogenesis of diabetic retinopathy: a 1990 update. Ophthalmology 98(5): 586-593.

7. Mandarino LJ (1992) Current hypotheses for the biochemical basis of diabetic retinopathy. Diabetes Care 15(12): 1892-1901.

8. Aiello L, Avery R, Arrigg P, Keyt B, Jampel H, et al. (1994) Vascular Endothelial Growth Factor In Ocular Fluid of Patients with Diabetic Retinopathy and Other Retinal Disorders. N Engl J Med 331(22):14801487.

9. Stitt AW, Li YM, Gardiner TA, Bucala R, Archer DB, et al. (1997) Advanced glycation end products (AGEs) co-localize with AGE receptors in the retinal vasculature of diabetic and of AGE-infused rats. Am J Pathol 150(2): 523-531.

10. Yamagishi $\mathrm{S}$, Amano $\mathrm{S}$, Inagaki $\mathrm{Y}$, Okamoto $\mathrm{T}$, Koga $\mathrm{K}$, et al. (2002) Advanced glycation end products-induced apoptosis and overexpression of vascular endothelial growth factor in bovine retinal pericytes. Biochem Biophys Res Commun 290(3): 973-978.

11.Yamagishi S, Imaizumi T (2005) Diabetic vascular complications: pathophysiology, biochemical basis and potential therapeutic strategy. Curr Pharm Des 11(18): 2279-2299.

12. Yamagishi S, Yonekura H, Yamamoto Y, Katsuno K, Sato F, et al. (1997) Advanced glycation end products-driven angiogenesis in vitro induction of the growth and tube formation of human microvascular 


\section{Current Research in Diabetes \& Obesity Journal}

endothelial cells through autocrine vascular endothelial growth factor J Biol Chem 272(13): 8723-8730.

13. Lu M, Perez VL, Ma N, Miyamoto K, Peng HB, et al. (1999) VEGF increases retinal vascular ICAM-1 expression in vivo. Invest Ophthalmol Vis Sci 40(8): 1808-1812.

14. Yamagishi S, Matsui T (2011) Advanced glycation end products (AGEs) oxidative stress and diabetic retinopathy. Curr Pharm Biotechno 12(3): 362-368.

15. Yamagishi S, Matsui T, Nakamura K, Takeuchi M, Imaizumi T (2006) Pigment epithelium-derived factor (PEDF) prevents diabetes-or advanced glycation end products (AGE)-elicited retinal leukostasis. Microvasc Res 72(1-2): 86-90.

16. Sharma K, Ziyadeh FN (1995) Hyperglycemia and diabetic kidney disease: the case for transforming growth factor- $\beta$ as a key mediator Diabetes 44(10): 1139-1146.

17. Dworkin L, Ichikawa I, Brenner B (1983) Hormonal modulation of glomerular function. Am J Physiol 244(2): F95-F104.

18. Kreisberg J, Venkatachalam M, Troyer D (1985) Contractile properties of cultured glomerular mesangial cells. Am J Physiol 249(4): F457-F463.

19. Takeuchi M, Takino J, Yamagishi S (2010) Involvement of the toxic AGEs (TAGE)-RAGE system in the pathogenesis of diabetic vascular complications: a novel therapeutic strategy. Curr Drug Targets 11(11): 1468-1482.

20. Myint KM, Yamamoto Y, Doi T, Kato I, Harashima A, et al. (2006) RAGE control of diabetic nephropathy in a mouse model. Diabetes 55(9): $2510-2522$

21. Yamamoto Y, Kato I, Doi T, Yonekura H, Ohashi S, et al. (2001) Development and prevention of advanced diabetic nephropathy in RAGE-over expressing mice. J Clin Invest 108(2): 261-268.

22. Matsui T, Yamagishi S, Ueda S, Nakamura K, Imaizumi T, et al. (2007) Telmisartan, an angiotensin II type 1 receptor blocker, inhibits advanced glycation end-product (AGE)-induced monocyte chemoattractant protein-1 expression in mesangial cells through downregulation of receptor for AGEs via peroxisome proliferator-activated receptor- $\gamma$ activation. J Int Med Res 35(4): 482-489.

23. Yamagishi S, Matsui T, Nakamura K, Inoue H, Takeuchi M, et al. (2008) Olmesartan blocks inflammatory reactions in endothelial cells evoked by advanced glycation end products by suppressing generation of reactive oxygen species. Ophthalmic Res 40(1): 10-15.

24. Dyck PJ, Giannini C (1996) Pathologic alterations in the diabetic neuropathies of humans: a review. J Neuropathol Exp Neurol 55(12): 1181-1193.

25. Yagihashi S (1995) Pathology and pathogenetic mechanisms of diabetic neuropathy. Diabetes Metab Rev 11(3): 193-225.

26. Thornalley PJ (2002) Glycation in diabetic neuropathy: characteristics, consequences, causes, and therapeutic options. Int Rev Neurobiol 50 37-57.

27. Wada R, Nishizawa Y, Yagihashi N, Takeuchi M, Ishikawa Y, et al. (2001) Effects of OPB-9195, anti-glycation agent, on experimental diabetic neuropathy. Eur J Clin Invest 31(6): 513-520.

28. Sekido H, Suzuki T, Jomori T, Takeuchi M, Yabe-Nishimura C, et al. (2004) Reduced cell replication and induction of apoptosis by advanced glycation end products in rat Schwann cells. Biochem Biophys Res Commun 320(1): 241-248.

29. Takeuchi M, Bucala R, Suzuki T, Ohkubo T, Yamazaki M, et al. (2000) Neurotoxicity of advanced glycation end-products for cultured cortical neurons. J Neuropathol Exp Neurol 59(12): 1094-1105.
30. Yamagishi S (2012) Potential clinical utility of advanced glycation end product cross-link breakers in age-and diabetes-associated disorders. Rejuvenation Res 15(6): 564-572.

31. Rizvi AA (2009) Cytokine biomarkers, endothelial inflammation, and atherosclerosis in the metabolic syndrome: emerging concepts. Am J Med Sci 338(4): 310-318.

32. Daffu G, del Pozo CH, O'Shea KM, Ananthakrishnan R, Ramasamy R, et al. (2013) Radical roles for RAGE in the pathogenesis of oxidative stress in cardiovascular diseases and beyond. Int J Mol Sci 14(10): 19891-19910.

33. Fukami K, Yamagishi S, Okuda S (2014) Role of AGEs-RAGE system in cardiovascular disease. Curr Pharm Des 20(14): 2395-2402.

34. Soro-Paavonen A, Zhang WZ, Venardos K, Coughlan MT, Harris E, et al. (2010) Advanced glycation end-products induce vascular dysfunction via resistance to nitric oxide and suppression of endothelial nitric oxide synthase. J Hypertens 28(4): 780-788.

35. Ando R, Ueda S, Yamagishi S, Miyazaki H, Kaida Y, et al. (2013) Involvement of advanced glycation end product-induced asymmetric dimethylarginine generation in endothelial dysfunction. Diab Vasc Dis Res 10(5): 436-441.

36. Ojima A, Ishibashi Y, Matsui T, Maeda S, Nishino Y, et al. (2013) Glucagon-Like Peptide-1 Receptor Agonist Inhibits Asymmetric Dimethylarginine Generation in the Kidney of Streptozotocin-Induced Diabetic Rats by Blocking Advanced Glycation End Product-Induced Protein Arginine Methyltranferase-1 Expression. Am J Pathol 182(1): 132-141.

37. Ueda S, Yamagishi S, Yokoro M, Okuda S (2014) Role of asymmetric dimethylarginine in cardiorenal syndrome. Curr Pharm Des 20(14): 2448-2455.

38. Cai W, He JC, Zhu L, Peppa M, Lu C, et al. (2004) High levels of dietary advanced glycation end products transform low-dersity lipoprotein into a potent redox-sensitive mitogen-activated protein kinase stimulant in diabetic patients. Circulation 110(3): 285-291.

39. Ishibashi Y, Matsui T, Takeuchi M, Yamagishi S (2011) Rosuvastatin blocks advanced glycation end products-elicited reduction of macrophage cholesterol efflux by suppressing NADPH oxidase activity via inhibition of geranylgeranylation of Rac-1. Horm Metab Res 43(9): 619-624.

40. Khera AV, Cuchel M, de la Llera-Moya M, Rodrigues A, Burke MF, et al. (2011) Cholesterol efflux capacity, high-density lipoprotein function, and atherosclerosis. N Engl J Med 364(2): 127-135.

41. Ishibashi Y, Matsui T, Fukami K, Ueda S, Okuda S, et al. (2015) Rivaroxaban inhibits oxidative and inflammatory reactions in advanced glycation end product-exposed tubular cells by blocking thrombin/ protease-activated receptor-2 system. Thromb Res 135(4): 770-773.

42. Ishibashi Y, Matsui T, Ueda S, Fukami K, Yamagishi S (2014) Advanced glycation end products potentiate citrated plasma-evoked oxidative and inflammatory reactions in endothelial cells by up-regulating protease-activated receptor-1 expression. Cardiovasc Diabetol 13: 60.

43. Ishibashi Y, Matsui T, Yamagishi S (2014) Apixaban exerts antiinflammatory effects in mesangial cells by blocking thrombin/ protease-activated receptor-1 system. Thromb Res 134(6): 13651367.

44. Yamagishi S, Fujimori H, Yonekura H, Yamamoto Y, Yamamoto H (1998) Advanced glycationendproducts inhibit prostacyclin production and induce plasminogen activator inhibitor-1 in human microvascular endothelial cells. Diabetologia 41(12): 1435-1441.

45. Yamagishi S, Yonekura H, Yamamoto Y, Fujimori H, Sakurai S, et al (1999) Vascular endothelial growth factor acts as a pericyte mitogen under hypoxic conditions. Lab Invest 79(4): 501-509. 


\section{Current Research in Diabetes \& Obesity Journal}

46. Hoenig MR, Bianchi C, Rosenzweig A, Sellke FW (2008) Decreased vascular repair and neovascularization with ageing: mechanisms and clinical relevance with an emphasis on hypoxia-inducible factor-1. Curr Mol Med 8(8): 754-767.

47. Chen Q Dong L, Wang L, Kang L, Xu B (2009) Advanced glycation end products impair function of late endothelial progenitor cells through effects on protein kinase Akt and cyclooxygenase-2. Biochem Biophys Res Commun 381(2): 192-197.

48. Bhatwadekar AD, Glenn JV, Li G, Curtis TM, Gardiner TA, et al (2008) Advanced glycation of fibronectin impairs vascular repair by endothelial progenitor cells: implications for vasodegeneration in diabetic retinopathy. Invest Ophthalmol Vis Sci 49(3): 1232-1241.

49. Suga T, Iso T, Shimizu T, Tanaka T, Yamagishi S, et al. (2011) Activation of receptor for advanced glycation end products induces osteogenic differentiation of vascular smooth muscle cells. J Atheroscler Thromb 18(8): 670-683.

50. Wu S, Song T, Zhou S, Liu Y, Chen G, et al. (2008) Involvement of $\mathrm{Na}^{+} / \mathrm{H}^{+}$ exchanger 1 in advanced glycation end products-induced proliferation of vascular smooth muscle cell. Biochem Biophys Res Commun 375(3): 384-389.

\section{Your next submission with Juniper Publishers will reach you the below assets}

- Quality Editorial service

- Swift Peer Review

- Reprints availability

- E-prints Service

- Manuscript Podcast for convenient understanding

- Global attainment for your research

- Manuscript accessibility in different formats

( Pdf, E-pub, Full Text, Audio)

- Unceasing customer service

Track the below URL for one-step submission https://juniperpublishers.com/online-submission.php 